\title{
The Lambda parameter and strange quark mass in two-flavour QCD
}

HU-EP-12/53

SFB-CPP-13-10

\author{
Patrick Fritzsch ${ }^{* \dagger}$ \\ Institut für Physik, \\ Humboldt-Universität zu Berlin, \\ Newtonstr. 15, 12489 Berlin, Germany \\ E-mail: fritzschaphysik.hu-berlin.de
}

\begin{abstract}
We present our final results for the Lambda parameter and strange quark mass of QCD with two dynamical quark flavors following the ALPHA collaboration's long-term strategy to compute fundamental parameters of QCD. This strategy includes non-perturbative renormalization and a recursive finite-size scaling procedure in the continuum to compute the renormalization group invariant quantities $\Lambda$ and $M_{\mathrm{s}}$. Here perturbation theory enters at scales of $\mathrm{O}(100 \mathrm{GeV})$ where it is well controlled. The last missing piece in this setup, namely the lattice scale has been recently determined via the kaon decay constant on large volume simulations $\left(m_{\pi} L \geq 4\right)$ made available through the Coordinated Lattice Simulations (CLS) effort.
\end{abstract}

Xth Quark Confinement and the Hadron Spectrum

8-12 October 2012

TUM Campus Garching, Munich, Germany

\footnotetext{
*Speaker.

${ }^{\dagger}$ in collaboration with F. Knechtli, B. Leder, M. Marinkovic, S. Schaefer, R. Sommer and F. Virotta, see [1]
} 
Even in QCD with $N_{\mathrm{f}}$ massless flavors there is a vast freedom in choosing a renormalization scheme and thus a definition for a renormalized strong coupling $\bar{g}_{s}^{2}(\mu)$ at some energy scale $\mu$. Due to dimensional transmutation each scheme has an intrinsic energy scale given by the Lambda parameter which is well-defined from the asymptotic behaviour of the coupling at hand:

$$
\Lambda=\lim _{\mu \rightarrow \infty} \mu\left[b_{0} \bar{g}_{s}^{2}(\mu)\right]^{-b_{1} /\left(2 b_{0}^{2}\right)} \exp \left[-1 /\left(2 b_{0} \bar{g}_{s}^{2}(\mu)\right)\right] .
$$

Hence, $\Lambda$ is a renormalization group invariant (RGI) and thus a fundamental parameter of the theory but trivially scheme-dependent; any two (well-behaved massless) schemes can be exactly related by the ratio of their Lambda parameters. Rather than fitting some experimental data within a scheme over a certain range of energies to extract $\Lambda$, the ALPHA collaboration traditionally follows a nonperturbative approach using lattice QCD simulations to solve the renormalization group equations behind eq. (1) in the continuum while keeping control over all systematic errors.

\section{Overall strategy to compute the Lambda parameter non-perturbatively}

In the present case of QCD with two degenerate dynamical quarks, the key equation reads

$$
\frac{\Lambda_{\overline{\mathrm{MS}}}^{(2)}}{f_{\mathrm{K}}}=\frac{1}{L_{1} f_{\mathrm{K}}} \times \Lambda_{\mathrm{SF}}^{(2)} L_{1} \times \frac{\Lambda_{\overline{\mathrm{MS}}}^{(2)}}{\Lambda_{\mathrm{SF}}^{(2)}} .
$$

Here $\Lambda_{\overline{\mathrm{MS}}}^{(2)}$ is given in units of the scale setting parameter $f_{\mathrm{K}}=155 \mathrm{MeV}$ (c.f. [2]), the kaon decay constant as physical input parameter. The ratio is decomposed into three different parts which have been determined independently. The ratio $\Lambda_{\overline{\mathrm{MS}}}^{(2)} / \Lambda_{\mathrm{SF}}^{(2)}=2.382035$ (3) [3] connects the $\overline{\mathrm{MS}}$ scheme to the Schrödinger Functional scheme (SF). The latter is used as massless intermediate finite-volume renormalization scheme to solve scale-dependent renormalization problems non-perturbatively using lattice QCD. In the SF the renormalization scale is given by the inverse box length, $\mu=1 / L$, which is implicitly fixed through a prescribed value of the non-perturbatively renormalized strong coupling $\bar{g}_{\mathrm{SF}}^{2}(L)$ at that scale. In our case it has been kept fix at $\bar{g}_{\mathrm{SF}}^{2}=4.484$, corresponding to a typical hadronic length scale $L=L_{1} \sim 0.4 \mathrm{fm}$ which allows to make contact to physical units in eq. (2). This fixed coupling is the starting point for the recursive finite-size scaling procedure in the SF scheme to connect hadronic and perturbative scales non-perturbatively. Using the data for the RG running from ref. [4] one gets $\Lambda_{\mathrm{SF}}^{(2)} L_{1}=0.264(15)$ in the continuum.

The missing and most expensive step in the computation of the Lambda parameter could recently be finished due to the combined CLS effort [5]. Computing $L_{1} f_{\mathrm{K}}$ actually means to perform the continuum extrapolation of $\left[L_{1} / a\right] \times\left[a f_{\mathrm{K}}\right]$ where $a$ is the lattice spacing and $a f_{\mathrm{K}}$ to be taken from lattice QCD simulations at the physical pion mass (w/o e.m. contributions). Since large volume lattice simulations with small lattice spacings at the physical pion mass are not yet state of the art in the lattice community, one still has to rely on a combined continuum and chiral extrapolation whose entangled systematic errors need to be well-controlled. On a set of CLS ensembles $\left(L m_{\mathrm{PS}} \geq 4, a<0.08 \mathrm{fm}, m_{\mathrm{PS}} \lesssim 500 \mathrm{MeV}\right)$ two complementary strategies for the (partially quenched) chiral extrapolation of the kaon decay constant have been used in ref. [1] to properly set the scale and attribute a systematic error. A subsequent continuum extrapolation finally gives $L_{1} f_{\mathrm{K}}=0.315(8)(2)$ that according to eq. (2) leads to

$$
\Lambda_{\overline{\mathrm{MS}}}^{(2)}=310(20) \mathrm{MeV} \text {. }
$$


Using the Sommer scale $r_{0}=0.503(10) \mathrm{fm}$ [1] we can unambiguously compare our estimate of $r_{0} \Lambda \frac{(2)}{\mathrm{MS}}=0.789(52)$ with those obtained from different methods by other lattice collaborations. Although the estimated error among the other results is comparable to ours, the absolute values are systematically smaller, i.e. $r_{0} \Lambda \frac{(2)}{\mathrm{MS}} \sim 0.59-0.72$ depending on the method used to extract the Lambda parameter. One main difference is that our method covers a wide range of scales up to $\mathrm{O}(100 \mathrm{GeV})$ where a matching to the perturbative running is known to be safe while for all other estimates perturbation theory already enters at $\mathrm{O}(1 \mathrm{GeV})$ where the situation is not that clear.

\section{Strategy for the computation of the strange quark mass}

We define the strange quark mass by the renormalized PCAC quark mass $\bar{m}_{\mathrm{s}}$ according to

$$
\frac{M_{\mathrm{s}}}{f_{\mathrm{K}}}=\frac{M}{\bar{m}\left(L_{1}\right)} \times \frac{\bar{m}_{\mathrm{s}}\left(L_{1}\right)}{f_{\mathrm{K}}} \quad \Rightarrow \quad \frac{\bar{m}_{\mathrm{s}} \overline{\mathrm{MS}}(\mu)}{f_{\mathrm{K}}}=\frac{\bar{m}^{\overline{\mathrm{MS}}}(\mu)}{M} \times \frac{M_{\mathrm{s}}}{f_{\mathrm{K}}} .
$$

The continuum factor $M / \bar{m}\left(L_{1}\right)=1.308$ (16) connects any renormalized quark mass in the SF at $\mu=1 / L_{1}$ to its RGI while $\bar{m}_{\mathrm{s}}^{\overline{\mathrm{MS}}}(\mu) / M$ connects the latter to a renormalized $\overline{\mathrm{MS}}$ mass at scale $\mu$. The bare PCAC strange quark mass is given through the requirement that $m_{\mathrm{K}}^{2} / f_{\mathrm{K}}^{2}$ attains its physical value at $m_{\mathrm{PS}}=m_{\pi}$. After taking the continuum limit and using two strategies for the chiral extrapolation to estimate a systematic error we get

$$
M_{\mathrm{S}}=138(3)(1) \mathrm{MeV}, \quad \bar{m}_{\mathrm{s}}^{\overline{\mathrm{MS}}}(2 \mathrm{GeV})=102(3)(1) \mathrm{MeV} .
$$

For all remaining details that had to be left out here we have to refer the interested reader to [1].

\section{Conclusions}

We summarized the strategy and results of the ALPHA collaboration to non-perturbatively estimate the Lambda parameter and strange quark mass in a model independent manner in $N_{\mathrm{f}}=2$ QCD [1]. The last step in this strategy that has recently been completed was the scale setting in lattice simulations of the CLS consortium. The value of $\Lambda \frac{(2)}{\mathrm{MS}}=320(20) \mathrm{MeV}$ is larger than the one obtained with a similar strategy in quenched QCD, 238(19) MeV, which reveals strong effects of the additional matter content and motivates us to extend this strategy to $N_{\mathrm{f}}>2$ where perturbation theory predicts a decrease with the number of flavors if the quark that is being decoupled is sufficiently heavy. The value reported for the strange quark mass is compatible within errors with the current PDG world average of $\overline{m_{\mathrm{s}}} \overline{\mathrm{MS}}(\mu=2 \mathrm{GeV})=95(5) \mathrm{MeV}$. However, it will be interesting to see how this compares in the future with a strange quark included in the sea sector. What still remains to be done in the two-flavor theory is the computation of $m_{\mathrm{u}, \mathrm{d}}$ and $m_{\mathrm{c}}$.

\section{Acknowledgments}

I would like to thank my colleagues F. Knechtli, B. Leder, M. Marinkovic, S. Schaefer, R. Sommer and F. Virotta for the many discussions and pleasant atmosphere during this collaboration.

\section{References}

[1] P. Fritzsch, F. Knechtli, B. Leder, M. Marinkovic, S. Schaefer, et al., Nucl.Phys. B865, 397 (2012).

[2] G. Colangelo, S. Dürr, A. Jüttner, L. Lellouch, H. Leutwyler, et al., Eur.Phys.J. C71, 1695, (2011).

[3] S. Sint and R. Sommer, Nucl.Phys. B465, 71 (1996).

[4] M. Della Morte et al., Nucl.Phys. B713, 378 (2005).

[5] “Coordinated Lattice Simulations,” https://twiki.cern.ch/twiki/bin/view/CLS/WebHome. 